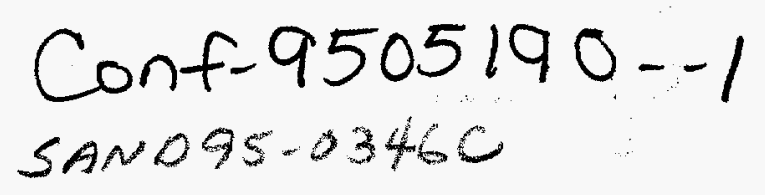

ADVANCED TECHNOLOGIES FOR PERIMETER INTRUSION DETECTION SENSORS

\section{J. D. Williams}

Sandia National Laboratories, United States of America*

\section{ABSTRACT}

Perimeter intrusion detection systems are an integral part of most physical security systems. Sandia National Laboratories, under the sponsorship of the U.S. Department of Energy, Office of Safeguards and Security; the U.S. Military Services; and many other U.S. Government Agencies, has over the last 20 years conducted surveys of available perimeter intrusion detection sensors and has tested many of the sensors manufactured in the United States and other countries. An overview of the newer and more advanced technologies employed in these sensors is provided.

\section{INTRODUCTION}

The development of integrated circuit fabrication techniques and the resulting devices have contributed more to the advancement of exterior intrusion detectors and alarm assessment devices than any other technology. The availability of this technology has led to the improvements in and further development of smaller more powerful computers, microprocessors, solid state memories, solid state cameras, thermal imagers, lowpower lasers, and shorter pulse width and higher frequency electronic circuitry. This paper presents information on planning a perimeter intrusion detection system, identifies the site characteristics that affect its performance, and describes improvements to perimeter intrusion detection sensors and assessment devices that have been achieved by using integrated circuit technology.

\section{PERIMETER INTRUSION DETECTION SYSTEM PLANNING}

The selection of intrusion detection equipment involves identifying the requirements and selecting the equipment and installation methods that best meet the overall system objectives. The system objectives, which include defining the purpose of the intrusion detection equipment and identifying the assumed threats, should quantify the requirements of the intrusion detection system in three primary areas: (1) the minimum acceptable probability of

${ }^{*}$ This work was supported by the United States Department of Energy under Contract DE-AC04-94AL85000. detecting the intruder $\mathrm{Pd}$, (2) the minimum allowable vulnerability of the equipment to defeat, and (3) the maximum allowable false/nuisance alarm rate (FAR/NAR). These parameters cannot be represented by single-value numbers; they are influenced by a large number of variables such as physical environment, weather, threat, maintenance, installation, regulations, procedures and operating personnel.

Site characteristics which affect the performance of perimeter sensors

The physical and environmental conditions that can affect exterior detection systems include topography, vegetation, wildlife, background noise, meteorological conditions, and types of soil and pavement. It is important to recognize that there is no "typical site" since combinations of conditions are site specific. Topographical concerns include slopes and hills, gullies and ditches, lakes, rivers and streams, swamps and temporary surface water, perimeter access points, and manmade structures. Vegetation includes all plant life such as trees, weeds, grass, bushes, and crop foliage. The vibration of the root systems of vegetation as well as the above ground motion of foliage can affect sensor performance. Wildlife of concern includes large and small animals, burrowing animals, and birds and insects. Background noise such as traffic, wind, natural and manmade seismic sources, and electromagnetic interference all must be taken into account. The specific type of meteorological information that may prove useful in the design and operation of sensor systems includes wind speed, temperature, rain, snow, hail, visibility, airborne corrosives, and electrical storms. Soil and pavement conditions primarily affect buried sensors.

No single sensor exists that will detect all types of intrusions with acceptably low FAR in normal outdoor environments. Single sensors can be employed to reliably detect the casual intruder who might simply climb a fence and walk through an area. However, in order to detect determined intruders while maintaining an acceptably low system NAR/FAR, appropriate combinations of sensor signals analyzed with 


\section{DISCLAIMER}

This report was prepared as an account of work sponsored by an agency of the United States Government. Neither the United States Government nor any agency thereof, nor any of their employees, make any warranty, express or implied, or assumes any legal liability or responsibility for the accuracy, completeness, or usefulness of any information, apparatus, product, or process disclosed, or represents that its use would not infringe privately owned rights. Reference herein to any specific commercial product, process, or service by trade name, trademark, manufacturer, or otherwise does not necessarily constitute or imply its endorsement, recommendation, or favoring by the United States Government or any agency thereof. The views and opinions of authors expressed herein do not necessarily state or reflect those of the United States Government or any agency thereof. 


\section{DISCLAIMER}

Portions of this document may be illegible in electronic image products. Images are produced from the best available original document. 
sophisticated sensor signal processing algorithms and the combination (fusion) of alarm outputs may be required.

\section{TECHNOLOGICAL TYPES OF SENSORS}

Intrusion detection sensors are usually categorized as either exterior or interior sensors. In this paper we are concerned only with exterior sensors. Exterior sensors include fence sensors, free-standing line sensors, buriedline sensors, wide-area sensors and point sensors. Exterior sensors can be further divided into several technological types. Another categorization of exterior sensors often used by military organizations is: tactical, portable, rapidly-deployable, semi-permanent, and fixed site. Within these categories is also a set of exterior sensors that are designated "unattended ground sensors (UGS)". The UGS systems are used in many military applications. There is also an increased interest in underground facilities. The sensors used for an underground facility may be different from those used for an above-ground facility. The basic sensing technologies used in all of these different designations are discussed below.

\section{General Exterior Sensors}

Exterior sensors can be further divided into technological types. Most of these technological types are covered in the following discussion.

Fence-associated sensors. Since it is desirable to detect an intruder as soon as possible, the first type of sensor encountered by an intruder is usually located at the outer perimeter of a facility. Fences, no matter how tall or how elaborate, offer little resistance to a determined intruder. Therefore, fence sensors can only help to form a viable perimeter delay system. However, even if a good perimeter system is employed it is only part of an overall security system and cannot be considered as complete detection. Fence-associated sensors offer no detection of an intruder who goes over or under the fence or who hides on the premises during a time when there is open access to the facility. The most common types of fence sensors are described in the following paragraphs.

Tilt-switch sensors consist of several series or parallelconnected switches coupled to a processor. Detection is based on the fact that any motion of the switch housing will result in the opening or closing of a contact switch or generate an output from a piezoelectric element. Switches are mounted on fence posts or fence fabric sections so that disturbance to the fence will cause switch action. Tilt switches offer detection of intrusion by sensing abnormal motions, shocks, or vibrations produced during attempts to climb over the fence, to penetrate the fence by cutting or burning, or to push or pull the fence up or down. Several different types of tilt-switch sensors are currently in use: mercury switch, pedestal-mounted ball, piezoelectric element, geophone, and reed switch. Most of the improvements in these types of sensors have been in the processors not in the sensing elements.

Strain-sensitive cable sensors are special cables that detect fence movement and/or possibly sounds. These cables provide coverage along the entire length of the fence to be protected. The cables "can be electret, triboelectric, magnetic or non-magnetic wire displacement in a dielectric casing, or fiber-optic (discussed in greater detail in a following section). The signal generated by the sensing cable is amplified and processed for frequency content, amplitude, and duration to allow differentiation between actual intrusions and environmental disturbances. Major environmental disturbances that cause the fence to be displaced or vibrate, thus causing nuisance alarms, are; wind, rain, and hail. Improvements in these types of sensors have been achieved by integration of weather stations, time domain reflectometers, microprocessors, and multiplexers into the processors to more accurately characterize intruders and minimize nuisance alarms. The system with the time domain reflectometer can be adjusted to compensate for differences in the condition of the fence and sensitivity of the cable to distances as short as one foot. Most of these improvements are only possible because of the increase in performance and reduction in size and cost realized with integrated circuitry and associated signal processing technology.

Taut-wire sensors, or wire-tension sensors consist of a series of wires installed under tension and attached to displacement sensors so that deflection of the wires produces a signal. The physical mechanism can be displacement of a magnet, displacement of the switch arm, or the electrical output of a strain gauge. Various wire configurations are possible, including free-standing and fence fabric mounted configurations.

Electric-field sensors can be fence mounted or freestanding. There are several volumetric electric-field sensors that consist of an alternating-current field generator that excites a field wire. One or more sensing wires couple into the resulting electric field, and an amplifier/signal processor detects changes in the signal characteristics of the sensing wires. Other electric-field devices process the signals collected from many wires. A signal is generated when a conductive body or a body with a high dielectric constant (such as a human body) distorts the field between the wires. Various portedcoaxial and other electric-field disturbance sensors are also available. The major improvements in these types of 
sensors are the introduction of digital signal processing, balanced phase designs, built-in test/calibration capabilities, and higher speed signal processing techniques. Lightning and manmade radio frequency signals can cause nuisance alarms on these types of sensors.

Fiber-optic sensors can be fence mounted and come in three varieties: 1.) those that detect vibration and changes in pressure, 2.) those that detect bending of the fiber, and 3.) those that detect when the fiber is broken or badly bent. A versatile type is one that detects vibration and changes in pressure. The sensor system consists of a fiber-optic cable and an electro-optic unit. The manufacture of the electro-optic unit is heavily dependent on integrated circuit processing technology. The fiberoptic cable is immune to electromagnetic interference and this is the major strength of fiber-optic sensors. Telemetry equipment, high voltage transmission lines, switching equipment, radar, or static discharge (such as from natural sources, i.e., lightning) does not affect this type of sensor.

Free-standing sensors. Free-standing sensors used for exterior applications include active and passive infrared, microwave, and electric-field sensors.

Active infrared sensors are free-standing line sensors that consist of infrared transmitters, photo-detectors, and application specific lenses. Several transmitters and receivers are usually employed to provide a multiple beam system. The beams are usually configured into a vertical infrared fence. A pulsed synchronous technique is used to reduce interference from and/or defeat by other sources of light. Another improvement in these types of sensors includes processing each beam that is broken to generate a profile of what caused the beams to be broken.

Passive infrared sensors. Every person, animal, or object emits infrared energy as a function of its surface temperature and size. Such radiated energy is in the wavelength region of 10 microns for normal ambient temperatures. Passive infrared (PIR) sensors detect changes in thermal infrared radiation within a specific field of view, responding to both changes of scene (including desired targets) and changes in illumination. PIR systems are widely used for interior applications, and are now being used for exterior free-standing and widearea applications.

Microwave sensors used for exterior applications can either be monostatic (transmitter and receiver are located in the same housing) or bistatic (transmitter and receiver are located in two separate housings). A bistatic microwave sensor uses a modulated transmitter and a receiver which are separated by a limited $(\cong 100$ meters) line-of-sight distance. Detection is based on the fact that an object moving through the beam will cause a signal change in the receiver. Major improvements in bistatic microwave sensors include spread spectrum technology, shorter pulse widths, printed circuit antennas, and better setup and diagnostic capabilities. An example of a new microwave sensor which incorporates many of these improvements is the Portable, Reconfigurable Line Sensor (PRLS). The PRLS is a bistatic sensor developed at Sandia for the US Air Force and is now commercially available. The PRLS can detect intruders ranging from a slow crawler (1 inch/second) to a high speed vehicle (70 $\mathrm{mph}$ ). One of the major attractions of the PRLS is the speed and ease of the set-up process. A single perimeter consisting of four PRLS units can be set up in about five minutes by unskilled personnel. The portability, ease of setup and operation, and RF alarm reporting capability makes the PRLS attractive for perimeter, portal, and gapfiller applications.

Electric-field sensors can also be free-standing. The operating principles were given in the fence sensor section.

Buried-line sensors. A number of different technologies have been used as buried-line sensors. Some of these included seismic, magnetic, seismic/magnetic, balanced pressure, ported coaxial, electret, and fiber optic. Presently the ported coaxial, and the fiber optic are the most popular. Because of the extra cost of installation, potential ground water problems, the possibility of cutting underground cables, and that the cables are not "relocatable" buried-line sensors are used only where they are absolutely necessary or in applications where covertness is important.

Fiber-optic sensors are a relatively new technology. The pressure sensitivity of the fiber-optic sensors, mentioned above in the fence sensor section, is also used in buriedline fiber-optic sensors. The buried-line fiber-optic sensor is not as popular as the fence mounted fiber-optic sensors.

Buried ported coaxial sensors are a type of electromagnetic volumetric sensor. There exists one pulsed ported coaxial sensor and four $\mathrm{CW}$ (i.e., continuous wave) ported coaxial sensors. All of these sensors generate an electromagnetic field between two buried parallel coaxial sensors constructed in such a way as to allow some of the energy from the transmit cable to enter corresponding openings in the receive cable, thus generating the field both above and below the ground. One such system a twin-lead cable (combining the 
transmit and receiver cables) which can be buried in one trench.

Wide-area sensors. Wide-area detection is becoming important in early warning applications, such as airplane parking areas and in other applications where an area is to be protected.

Passive infrared sensors are used to automatically detect human intruders over wide areas. Some of these sensors use imaging and video motion detection techniques. Such a capability can provide early warning beyond the perimeter at fixed sites, and can also be used for portable security around mobile assets. Sandia has been working on automatic detection systems based on the thermal contrast and motion of human intruders for several years, and has found that detection is sometimes difficult, depending on solar and other environmental conditions. Solar heating can dominate human thermal radiation by 100 fold, and dynamic background temperature changes can limit detector sensitivity.

Monostatic microwave sensors have the transmitting and receiving antenna co-located and the functions of sending and receiving often share a single antenna. Intrusion detection can be based on the frequency shift between the transmitted and received signal caused by the Doppler effect from a moving object in the beam or it can be based on other field disturbances. The shape of the detection zone is governed by the characteristics of the antenna but is roughly similar to an elongated balloon. Monostatic microwave sensors can be range gated to prevent detection outside the desired envelope. They can have antennas which produce narrow or wide-area coverage.

First generation video-motion detection products were based on analog technology and could not always discriminate between real alarm conditions and normal picture changes. Analog motion detection depends on level changes within the active video. The development of digital processing produced tremendous improvements in video motion detection. Digital processing breaks up the video into individual points (pixels), which greatly increases the accuracy of video motion detection.

Acoustic arrays have been used in airborne vehicle detection applications and wide-area detection. Microphones can be arranged in a variety of arrays so that the direction and range of the acoustic source can be estimated and tracked by proper signal processing.

Point sensors. Point sensors are those which provide a small zone of coverage and can be used as "gap fillers" and as tamper or "weak-link" protection devices. Point sensors can be geophones, passive infrared, electromagnetic (field disturbance) point sensors, Doppler radar sensors or many of the sensors mentioned for wide-area applications (usually with decreased sensitivity). Field disturbance sensors are often used in automobile burglar alarms.

\section{ALARM PROCESSING}

In most of the sections above it has been mentioned that the basic sensing technologies have not changed significantly, but that great changes had been made in the signal processors. Early sensor processors used a threshold detector to produce an alarm when the amplitude of an analog signal exceeded a predetermined value. With smaller, more powerful computers and solidstate memories, more sophisticated signal (alarm) processing is now possible. In addition to all the techniques mentioned above, signal comparison and analysis using Fast Fourier Transforms (FFT's) and other techniques are used to extract more information from the raw sensor signals than was ever possible using simple threshold detection techniques.

\section{SENSOR FUSION}

For many years it has been the vision of security professionals that if many sensors of different technological types could be employed and their outputs properly combined (fused), the type of intruder could be determined and false/nuisance alarms could be minimized without other means of alarm assessment. Early attempts to realize the vision included use of digital logic circuitry, and weighted voting. Voting weights are assigned based on measured weather parameters. These techniques were only partly successful. Today a number of new techniques using fuzzy logic, neural networks, adaptive learning and complex computer algorithms help to realize the vision. Several of these techniques are working well and even more improvements are expected soon. One robot system uses a programmable fuzzy logic certainty engine to weigh multiple variables, create a composite picture from data supplied by an array of sensors, and perform real-time threat identification and assessment onboard the vehicle. All of these depend almost entirely on the advances that have been realized by fully utilizing integrated circuit processing techniques.

\section{ALARM ASSESSMENT}

Automation and systems integration are just two advanced technologies that are experiencing wide acceptance. It is expected that integrated security systems will be handling intrusion detection alarms, building automation, mechanical and electronic access control, fire 
alarms, electronic article surveillance, video alarm assessment, computer security and even patrol/guard service management all within one powerful database. Personal computers have evolved to the point where large mainframe computers are no longer necessary. The sophistication and power of personal computers are allowing manufacturers to develop systems that no longer require video time-lapse recorders, and monitors, but in their place are using computer memory storage and computer screen display. Also, microprocessor-based matrix switchers interface with alarm signals while monitoring systems with hundreds of cameras. The integrated systems can transmit near real-time video signals through telephone lines or wireless media. Such integrated systems put video alarm assessment, and access control at the fingertips of security personnel. The operations of these systems are simple to use with mouse, touch screen, or voice control technology.

\section{Video systems}

Asymmetric video, using telephone line transmission, overcomes the limitations of distance. As a modem can communicate with a computer located in any part of the world, video telephone line systems can receive transmissions from any installation with a telephone line. Previously the problem with this type of technology was the speed of transmission. Telephone lines are limited to a data rate of 9600 baud, a data transmission speed far below the rates required for real time video. By processing the signal in digital form, instead of analog, more information is transmitted. While the data rate of the telephone lines remains at 9600 baud, the digital system reads the action within a picture and only transmits the changes. The process works in the following way. Upon start up, the system reads the first frame of video and establishes it as a key frame. This process takes about ten seconds. After this only the changes in the picture are transmitted. If no changes occur, the picture to picture transmission is very rapid, but if many changes occur the transmission becomes longer but in no case longer than the original picture. This type of video processing is known as asymmetrical video. The system uses a computer and provides several advantages over former systems. One advantage is better picture quality. Video is recovered using computer image processing methods. In addition, changes can often be made just by issuing new software, without the need to make any equipment hardware changes.

In addition to the video, alarm signals issued at the site can be use to dial up a computer receiver, begin the transmission and even instruct a recorder at the receiver site to begin recording. Images can be saved on a hard disk and recalled. With the use of digital signals, video telephone line transmission moved from poor quality transmission to a quality level useful for security systems. Increased computer power has resulted in the ability to display four individual pictures on a single screen making the system more efficient.

Great improvements have recently been achieved in miniaturization (very small cameras are now available), light sensitivity (down to $0.1 \mathrm{lux}$ ) and higher resolution. Pre-alarm video assessment is also aiding in the alarm assessment problem. Pre-alarm video is accomplished by continuously recording, then saving the portion of the video that occurred just prior to an alarm occurring.

\section{Image processing.}

A number of different image processing technologies have been or are being developed. They include integration of image processing with other intrusion detection systems, video motion detection and object recognition. Ways of "looking beyond" the perimeter includes a number of range imaging technologies such as laser and synthetic aperture radar imaging. In addition to ground based warning systems, airborne early warning systems are being developed.

\section{Tracking}

Video motion detectors and other image processing algorithms can provide tracking information by providing a shaded trace of previous motion. Since thermal imagers operate with conventional video signals, tracking is possible for day and night situations independent of whether the area being viewed is lighted or unlighted.

Tracking techniques of persons who carry global positioning system (GPS) receivers or other active and passive devices are also possible. Since these usually require the person being tracked to obtain a badge or other device, such tracking is used mostly for monitoring security personnel or those authorized to be within the area of interest.

\section{CONCLUSION}

The most important technological advance in exterior intrusion detectors and alarm assessment devices during recent years has been in digital signal processing, but such processing was not practical until the development of high density integrated circuits and their associated fabrication techniques. Until new sensing technologies are developed it is expected that future advances in exterior intrusion detectors and alarm assessment devices will continue to depend on digital signal processing and integrated circuit fabrication techniques. 